\title{
Some similarity states of stably- stratified homogeneous turbulence
}

\author{
By J. R. Chasnov
}

The Hong Kong University of Science and Technology, Clear Water Bay, Kowloon, Hong Kong

The decay of statistically homogeneous velocity and density fluctuations in a stablystratified fluid is considered. Over decay times long compared to the turbulence timescale but short compared to the period of internal gravity waves, three distinct high Reynolds number similarity states may develop. These similarity states are a consequence of the invariance of the low wavenumber coefficients of the three-dimensional kinetic or potential energy spectrum; and their preferential development depends on the relative magnitudes of the initial kinetic and potential energy per unit mass of the fluid. When the turbulence has decayed over a time comparable to the period of the gravity waves, the three similarity states mentioned above are disrupted. Evidence will be presented of a new similarity state which then develops asymptotically. In this similarity state, the time decay exponent of the total energy per unit mass of the turbulence is reduced by a factor of two from its value for decaying isotropic turbulence, and the associated vertical integral scale approaches a constant independent of time.

\section{Introduction}

The statistics of homogeneous turbulence in fluids of infinite extent typically depend on time throughout their entire evolution. In homogeneous turbulence at high Reynolds numbers, similarity states of the flow field may replace the statistically stationary states that typically occur in bounded flows. In these similarity states the turbulence spectrum decays without change of shape so that in an appropriately scaled coordinate system the spectrum is independent of time. Some of the homogeneous flow fields for which similarity states have been observed by large-eddy simulation include decaying isotropic turbulence, passive scalars transported by isotropic turbulence with or without a uniform mean gradient, and buoyancy-generated turbulence. The existence of a similarity state for decaying homogeneous isotropic turbulence was postulated early on (Kolmogorov, 1941) as was that for a transported homogeneous isotropic passive scalar field (Corrsin, 1951). More recent work (Batchelor, Canuto \& Chasnov, 1992; Chasnov, 1994) indicates that hitherto unsuspected similarity states of homogeneous turbulence may exist for non-isotropic flows which contain more complicating physics, such as flows with buoyancy forces and uniform passive scalar gradients.

It is a natural extension of our earlier work in buoyancy-generated turbulence and turbulence with uniform passive scalar gradients to consider whether high Reynolds number similarity states exist for homogeneous turbulence in a stably-stratified fluid with both buoyancy effects and active scalar (density) gradients. In this paper, we first show how 
some of the flows previously considered can occur in a stably-stratified fluid at large Froude numbers. We will also present some analytical arguments and numerical results which provide evidence for a new similarity state which develops at small Froude numbers.

\section{The governing equations}

Choosing our co-ordinate system such that the $z$-axis is pointed vertically upwards, we assume a stable density distribution $\rho=\rho_{0}-\beta z+\rho^{\prime}$, where $\rho_{0}$ is a constant, uniform reference density, $\beta>0$ is a constant, uniform density gradient along $z$, and $\rho^{\prime}$ is the density deviation from the horizontal average. The kinematic viscosity $\nu$ and molecular diffusivity $D$ of the fluid are assumed constant and uniform. After application of the wellknown Boussinesq approximation, the governing equations for the fluid velocity $\mathbf{u}$ with zero mean and the density fluctuation $\rho^{\prime}$ are

$$
\begin{gathered}
\nabla \cdot \mathbf{u}=0 \\
\frac{\partial \mathbf{u}}{\partial t}+\mathbf{u} \cdot \nabla \mathbf{u}=\frac{\rho^{\prime} \mathbf{g}}{\rho_{\mathbf{0}}}-\frac{\nabla\left(p+\rho_{0} g z\right)}{\rho_{0}}+\nu \nabla^{2} \mathbf{u} \\
\frac{\partial \rho^{\prime}}{\partial t}+\mathbf{u} \cdot \nabla \rho^{\prime}=\beta u_{3}+D \nabla^{2} \rho^{\prime}
\end{gathered}
$$

where $\mathbf{g}=-\mathbf{j} g$ with $g>0, \mathbf{j}$ is the vertical (upwards) unit vector, and $p$ is the fluid pressure.

Our earlier work considered various limiting forms of (2.1)-(2.3) for which one of $g$ or $\beta$ was taken equal to zero. By a suitable non-dimensionalization of Eqs. (2.1) - (2.3), we will show that under certain conditions the terms containing $g$ and $\beta$ may also be negligible in a stably-stratified fluid. It is convenient to define a normalized density fluctuation $\theta$ such that it has units of velocity, $\theta=\sqrt{g / \rho_{0} \beta} \rho^{\prime}$. Use of $\theta$ instead of $\rho^{\prime}$ in $(2.2)-(2.3)$ modifies the terms proportional to $g$ and $\beta$ into terms proportional to $N$, where $N=\sqrt{g \beta / \rho_{0}}$ is the Brunt-Väisälä frequency associated with the internal waves of the stably stratified flow. The mean-square statistics $\frac{1}{2}\left\langle\mathbf{u}^{2}\right\rangle$ and $\frac{1}{2}\left\langle\theta^{2}\right\rangle$ are the kinetic and potential energy of the fluid per unit mass, respectively. The equations of motion conserve the total energy per unit mass in the absence of viscous and diffusive dissipation.

Now, defining dimensionless variables as

$$
T=t \frac{u_{0}}{l_{0}}, \quad X=\frac{x}{l_{0}}, \quad \mathbf{U}=\frac{\mathbf{u}}{u_{0}}, \quad P=\frac{\left(p+\rho_{0} g z\right)}{\rho_{0} u_{0}^{2}}, \quad \Theta=\frac{\theta}{\theta_{0}},
$$

where $l_{0}, u_{0}$ and $\theta_{0}$ are as yet unspecified length, velocity, and normalized density scales, the equations of motion (2.1)-(2.3) become

$$
\begin{gathered}
\nabla \cdot \mathbf{U}=0, \\
\frac{\partial \mathbf{U}}{\partial T}+\mathbf{U} \cdot \nabla \mathbf{U}=-\mathbf{j} \frac{1}{F_{0}} \frac{\theta_{0}}{u_{0}} \Theta-\nabla P+\frac{1}{R_{0}} \nabla^{2} \mathbf{U}, \\
\frac{\partial \Theta}{\partial T}+\mathbf{U} \cdot \nabla \Theta=\frac{1}{F_{0}} \frac{u_{0}}{\theta_{0}} U_{3}+\frac{1}{\sigma R_{0}} \nabla^{2} \Theta,
\end{gathered}
$$


where

$$
F_{0}=\frac{u_{0}}{N l_{0}}, \quad R_{0}=\frac{u_{0} l_{0}}{\nu}, \quad \sigma=\frac{\nu}{D}
$$

The dimensionless groups $F_{0}$ and $R_{0}$ can be regarded as initial Froude and Reynolds numbers of the flow, respectively, although their precise definition is yet dependent on our specification of $l_{0}, u_{0}$, and $\theta_{0} ; \sigma$ is the Schmidt (or Prandtl) number of the fluid.

\section{Three large Froude number flows}

We show here that particular initial fluctuating velocity and density fields in a stablystratified fluid can result in the establishment of distinctly different flows when the initial Froude number of the turbulence is large. The important point here is that with wellchosen initial flow fields either or both of the source/sink terms, (those terms proportional to the inverse Froude number in (2.6) and (2.7)), may be negligible over long times.

Flow 1: Isotropic turbulence transporting an isotropic passive scalar

We consider an initial generation of isotropic velocity and density fields of comparable integral scales and kinetic and potential energies. We identify the unspecified length scale $l_{0}$ with the initial integral scale of the flow, and $u_{0}$ and $\theta_{0}$ with the initial root-mean-square values of the velocity and normalized density fluctuations, respectively. If $F_{0} \gg 1$, both of the terms multiplied by $1 / F_{0}$ in (2.6) and (2.7) are small initially. Over times in which these terms remain small, the velocity fluctuations decouple from the density field and the turbulence decays isotropically while transporting an isotropic passive scalar field.

Flow 2: Isotropic turbulence in a passive scalar gradient

Here, we envision the generation of an initial isotropic velocity field with given kinetic energy and integral length scale, and no initial density fluctuations. We identify $l_{0}$ and $u_{0}$ as in flow 1. However, the initial conditions introduce no intrinsic density scale. So that $\Theta$ attains a value close to unity, we set the dimensionless group multiplying $U_{3}$ in (2.7) equal to one, yielding $\theta_{0}=N l_{0}$. The dimensionless group multiplying $\Theta$ in (2.6) then becomes $1 / F_{0}^{2}$, so that if $F_{0} \gg 1$, this term is small initially. As long as it remains small, the generated density fluctuations are passive and the resulting equations govern the evolution of decaying isotropic turbulence in the presence of a mean passive scalar gradient.

\section{Flow 3: Buoyancy-generated turbulence}

The fluid is assumed to be initially at rest with some given random density distribution. We identify $l_{0}$ and $\theta_{0}$ with the initial integral scale and root-mean-square value of the $\theta$ field, respectively. So that $U$ attains a value of order unity, we set the dimensionless group multiplying $\Theta$ in equation (2.6) equal to one, yielding $u_{0}=\sqrt{N l_{0} \theta_{0}}$. The dimensionless group multiplying $U_{3}$ in $(2.7)$ is now equal to $1 / F_{0}^{2}$, so that if $F_{0} \gg 1$ this term is small at the initial instant. Over times for which this term remains small, the resulting equations govern the evolution of buoyancy-generated turbulence.

How long do the above flows evolve before the effects of the neglected terms become important? Consider the evolution equations (2.6) and (2.7) after the flow fields have evolved over a time $t$. The relevant length, velocity, and normalized density scale of the flow are now those which characterize the fields at time $t$. The source/sink terms are of the 
same order when the velocity and normalized density scales are of comparable magnitude. This condition is satisfied by flow 1 from the initial instant. However, the density scale $\theta^{\prime}$ and velocity scale $u^{\prime}$ at time $t$ in flows 2 and 3 , respectively, increase from initial values of zero and can be estimated as $\theta^{\prime} \propto N u^{\prime} t$ in flow 2, and $u^{\prime} \propto N \theta^{\prime} t$ in flow 3 . Hence, the density and velocity scales become comparable when $t>1 / N$. Also, the Froude number of the flow at time $t$ can be shown to be proportional to $1 / N t$, so that it also becomes small when $t>1 / N$.

We have thus arrived at the intuitive result that physical effects associated with internal gravity waves affect the flow dynamics only after an evolution time comparable to the period of the gravity waves. For flows of initially large Froude number, the velocity and density fields evolve over many turbulence time scales before internal waves become dynamically important. Hence, similarity states associated with each of the above flow regimes may be established before a significant decrease in the flow Froude number. In the next Section, we briefly review the salient features of these similarity states.

\section{Asymptotic similarity states at large Froude numbers}

The similarity states which develop in the above flows depend on the form of the kinetic and potential energy spectra at low wavenumbers. Defining the kinetic energy spectrum $E_{k}(k, t)$ and the potential energy spectrum $E_{p}(k, t)$ to be the spherically-integrated three dimensional Fourier transform of $\frac{1}{2}\left\langle u_{i}(\mathbf{x}, t) u_{i}(\mathbf{x}+\mathbf{r}, t)\right\rangle$ and $\frac{1}{2}\langle\theta(\mathbf{x}, t) \theta(\mathbf{x}+\mathbf{r}, t)\rangle$, respectively, we write an asymptotic expansion of the spectra near $k=0$ as

$$
E_{k}(k, t)=2 \pi k^{2}\left(B_{0}+B_{2} k^{2}+\ldots\right), \quad E_{p}(k, t)=2 \pi k^{2}\left(C_{0}+C_{2} k^{2}+\ldots\right),
$$

where $B_{0}, B_{2}, \ldots$, and $C_{0}, C_{2}, \ldots$ are the lowest-order coefficients of the expansion. For brevity, we consider here only flow fields for which $B_{0}$ and $C_{0}$ are non-zero unless the entire spectrum is zero. The invariance of one or both of these low wavenumber coefficients lead directly to the establishment of different similarity states.

\section{Flow 1: Isotropic turbulence transporting an isotropic passive scalar}

The low wavenumber coefficients $B_{0}$ and $C_{0}$ are separately invariant (Saffman, 1967a; Corrsin, 1951), and when they are non-zero the high-Reynolds number asymptotic results for the kinetic energy, scalar-variance and integral scale may be determined by dimensional analysis to be (Saffman, 1967b; Larcheveque, et al., 1980)

$$
\left\langle\mathbf{u}^{2}\right\rangle \propto B_{0}^{\frac{2}{5}} t^{-\frac{6}{5}}, \quad\left\langle\theta^{2}\right\rangle \propto C_{0} B_{0}^{-\frac{3}{5}} t^{-\frac{6}{5}}, \quad l \propto B_{0}^{\frac{1}{5}} t^{\frac{2}{5}} .
$$

The nature of this similarity state is such that the kinetic and potential energy spectra decay without change of shape so that stationary spectra may be defined by the appropriate scaling of the wavenumber and spectral amplitudes.

Flow 2: Isotropic turbulence in a passive scalar gradient

The passive density (scalar) fluctuations for this flow are generated by velocity fluctuations along the direction of the mean gradient; consequently, the low wavenumber coefficient of the potential energy spectrum is no longer invariant in time. Rather, $C_{0}$ depends directly on the invariant $B_{0}, N$ and $t$ by

$$
C_{0}(t)=\frac{1}{3} N^{2} B_{0} t^{2}
$$


Use of (4.3) in (4.2) yields the asymptotic growth of the scalar variance (Chasnov, 1994)

$$
\left\langle\theta^{2}\right\rangle \propto N^{2} B_{0}^{\frac{2}{5}} t^{\frac{4}{5}} .
$$

Flow 3: Buoyancy-generated turbulence

Here, the velocity fluctuations are generated by density fluctuations and $B_{0}$ is no longer invariant; however $C_{0}$ is invariant. The coefficient $B_{0}$ is related to $C_{0}, N$ and $t$ by

$$
B_{0}(t)=\frac{2}{3} N^{2} C_{0} t^{2}
$$

and substituting (4.5) into (4.2) yields (Batchelor, et al., 1992)

$$
\left\langle\mathbf{u}^{2}\right\rangle \propto\left(N^{2} C_{0}\right)^{\frac{2}{5}} t^{-\frac{2}{5}}, \quad\left\langle\theta^{2}\right\rangle \propto C_{0}\left(N^{2} C_{0}\right)^{-\frac{3}{5}} t^{-\frac{12}{5}}, \quad l \propto\left(N^{2} C_{0}\right)^{\frac{1}{5}} t^{\frac{4}{5}} .
$$

An interesting and unusual feature of the similarity state for buoyancy-generated turbulence is an increase in the flow Reynolds number asymptotically.

\section{The flow at small Froude numbers}

After the stratified flow evolves over a time $t \sim 1 / N$, the Froude number is of order unity so that the above large Froude number similarity states are no longer valid. As the flow evolves further so that $N t \gg 1$, the Froude number may be expected to become small, and it is of some interest to consider whether a different similarity state of the flow field is established asymptotically provided the Reynolds number of the flow remains large. To construct a similarity state, an invariant of the kinetic and potential energy spectra near $k=0$ must be determined. Such an invariant does indeed exist and is associated with the low wavenumber coefficient of the total energy spectrum $E(k)$, defined by $E(k)=E_{k}(k)+E_{p}(k)$. An expansion of the total energy spectrum near $k=0$ yields

$$
E(k, t)=2 \pi k^{2}\left(A_{0}+A_{2} k^{2}+\ldots\right),
$$

where $A_{0}=B_{0}+C_{0}$ and $A_{2}=B_{2}+C_{2}$ are the sum of the low-wavenumber kinetic and potential energy spectral coefficients. The coefficient $A_{0}$ can be shown to be an exact invariant of the flow. Here, we consider its value to be non-zero at the initial instant.

We thus have a new invariant $A_{0}$ upon which to base an asymptotic similarity state. However, a straightforward dimensional analysis is now complicated by the addition of another relevant dimensionless group, namely $N t$, which is directly proportional to the number of wave periods over which the flow has evolved. For asymptotically large Froude number flows, $N t$ is vanishingly small and does not enter into the scalings; however, this may not be the case at small Froude numbers when $N t$ is large.

Despite the above difficulty, we nevertheless attempt a dimensional analysis of this problem by considering the evolution of the total energy of the flow, $e=\left(\left\langle\mathbf{u}^{2}\right\rangle+\left\langle\theta^{2}\right\rangle\right) / 2$, for which there exists the associated invariant $A_{0}$. Assuming a power law dependence on time, dimensional analysis then yields

$$
e=c_{e} A_{0}^{\frac{2}{5}} t^{-\frac{6}{5}}(N t)^{x}
$$


where we write the proportionality constant $c_{e}$ explicitely, and $x$ is an unknown exponent.

In addition to the total energy decay, it is of interest to consider the evolution of the integral scales of the flow field associated with the total energy. The integral scales may evolve differently depending on whether they are measured parallel or perpendicular to the vertical axis. Defining the vertical integral scale of the total energy to be $l_{v}$, and that of the horizontal integral scale to be $l_{h}$, dimensional analysis yields

$$
l_{v}=c_{l_{v}} A_{0}^{\frac{1}{5}} t^{\frac{2}{5}}(N t)^{y}, \quad l_{h}=c_{l_{h}} A_{0}^{\frac{1}{5}} t^{\frac{2}{5}}(N t)^{z},
$$

where $c_{l_{v}}$ and $c_{l_{h}}$ are proportionality constants, and $y$ and $z$ are two additional exponents.

A consideration of the total energy spectrum (5.1) as a function of the wavenumber magnitude $k$ is not entirely appropriate for a flow in which the time dependencies of the integral scales in the vertical and horizontal directions may be different. Rather, one should define an energy spectrum as an explicit function of the vertical and horizontal wavenumbers. A simple argument based on the existence of the invariant $A_{0}$ and an assumption of a self-similar decay in time of this redefined energy spectrum yields the constraint

$$
e l_{v} l_{h}^{2} \propto A_{0} ; \quad \text { or equivalently, } \quad x+y+2 z=0 .
$$

In addition, a heuristic argument can be offered which determines the unknown exponent $x$. At small Froude numbers, two disparate timescales of the flow exist: the fast timescale of the wave field and the slow time scale of the turbulence. If we assume that the correlation time of the nonlinear transfer is directly proportional to the fast time scale $1 / N$, then dimensionally

$$
\frac{d e}{d t}=\frac{1}{N} A_{0}^{-\frac{2}{3}} e^{\frac{8}{3}}
$$

which may be integrated directly to yield the value $x=3 / 5$ in $(5.2)$.

We do not yet have have an a priori argument to determine the remaining unknown exponents $y$ and $z$, although these exponents must satisfy the constraint given by (5.4). Rather, in the next Section we present the results of large-eddy simulations in which estimates for all three exponents may be computed.

\section{Large-eddy simulations at small Froude numbers}

To obtain a high Reynolds number flow at small Froude numbers, we perform large-eddy simulations of Eqs. (2.1) - (2.3) using a pseudo-spectral code for homogeneous turbulence (Rogallo, 1981). For the subgrid scale model, we employ a spectral eddy-viscosity and eddy-diffusivity similar to that of Chollet and Lesieur (1981). We take the initial kinetic energy spectrum of the form given by Chasnov (1994) with the low wavenumber portion of the spectrum proportional to $k^{2}$. The initial potential energy spectrum is taken to be zero. In the large Froude number regime, this corresponds to flow 2 above: isotropic turbulence in a passive scalar gradient. Preliminary calculations showed that the horizontal integral scales grew more rapidly than the vertical scales, in agreement with previous direct numerical simulation results (Riley, et al., 1981), and that it was optimal to use a computational box which was eight times longer in the horizontal directions than in the vertical. Accordingly, we took a computational box length of $4 \pi$ in the two horizontal 

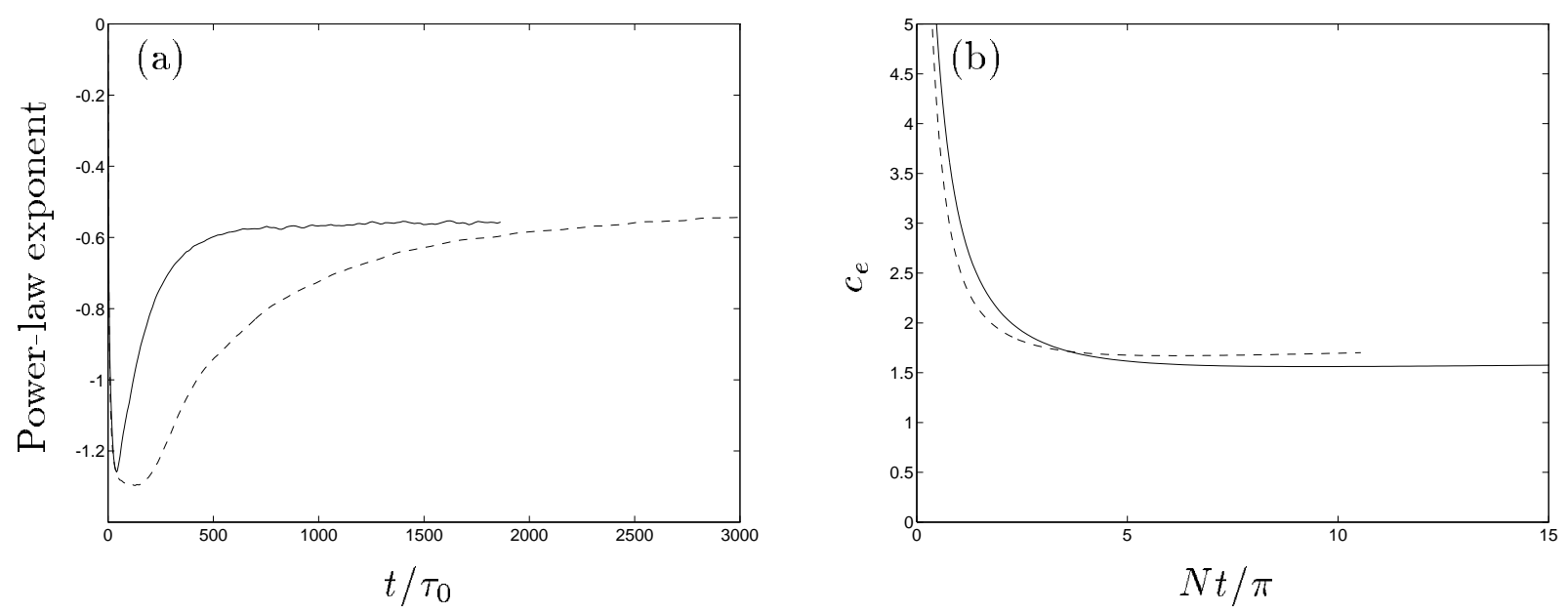

Figure 1. The energy statistics:,$- F_{0}=16.5 ; \cdots, F_{0}=93.2$; (a) time-evolution of the power-law exponent of $e$; (b) verification of the scaling relation given by (5.3) with $x=3 / 5$.

directions and $\pi / 2$ in the vertical direction, with a corresponding grid resolution of $512 \times$ $512 \times 64$ so that the grid remained cubic at the smallest resolved scales. With a periodic box, the vertical wavenumber then took the values $k_{z}=0, \pm 4, \pm 8, \ldots, \pm 128$ and the horizontal wavenumbers took the values $k_{x}, k_{y}=0, \pm 1 / 2, \pm 1, \ldots, \pm 128$. The peak of the initial isotropic kinetic energy spectrum $k_{p}$ was placed at a wavenumber of 64 . Two computations were performed with initial Froude number $F_{0}=u_{0} / N l_{0}$ given by $F_{0}=16.5$ and 93.2. In the definition of $F_{0}, u_{0}$ is taken as the initial root-mean square velocity fluctuation, and $l_{0}=\sqrt{\pi} / k_{p}$. The computations were performed holding $u_{0}$ and $l_{0}$ fixed and varying $N$ by a factor of approximately 5.65. The results of the computation are used here to test the postulated scalings given in $\S 5$ and to compute values of the unknown exponents $x, y$, and $z$.

In Figure 1a, we plot the power-law exponent of $t$ (i.e., the logarithmic derivative with respect to $t$ ) of the total energy as a function of $t / \tau_{0}$ for both initial Froude number flows, where $\tau_{0}=l_{0} / u_{0}$. The asymptotic value of the time exponent is approximately $-3 / 5$, indicating a value of $x$ in (5.2) equal to $3 / 5$, in agreement with our heuristic argument. In figure $1 \mathrm{~b}$, we plot the proportionality constant $c_{e}$ of (5.2), i.e., we plot the evolution of $e /\left(A_{0}^{2 / 5} N^{3 / 5} t^{-3 / 5}\right)$, for both initial Froude number flows. The approximate convergence of the two curves at large values of $N t$ to a constant confirms the overall scaling given by (5.2) with $x=3 / 5$ and $c_{e} \approx 1.6$.

The vertical and horizontal integral scales of the total energy are plotted versus $t / \tau_{0}$ in Figure 2a for both initial Froude number flows. Evidently, at large-times the vertical integral scale approached a constant, independent of time. This implies that $y=-2 / 5$ in (5.3). The additional constraint of (5.4) then yields $z=-1 / 10$. In Figure 2b, we plot the evolution of $c_{l_{v}}$ and $c_{l_{h}}$ for both flows. Again the simulations are in reasonable agreement with the overall scaling given by (5.3), with $c_{l_{v}} \approx 1.4$ and $c_{l_{h}} \approx 1.6$.

We have thus presented findings of a new similarity state which develops at large Reynolds numbers and small Froude numbers when the initial flow field consists of an 

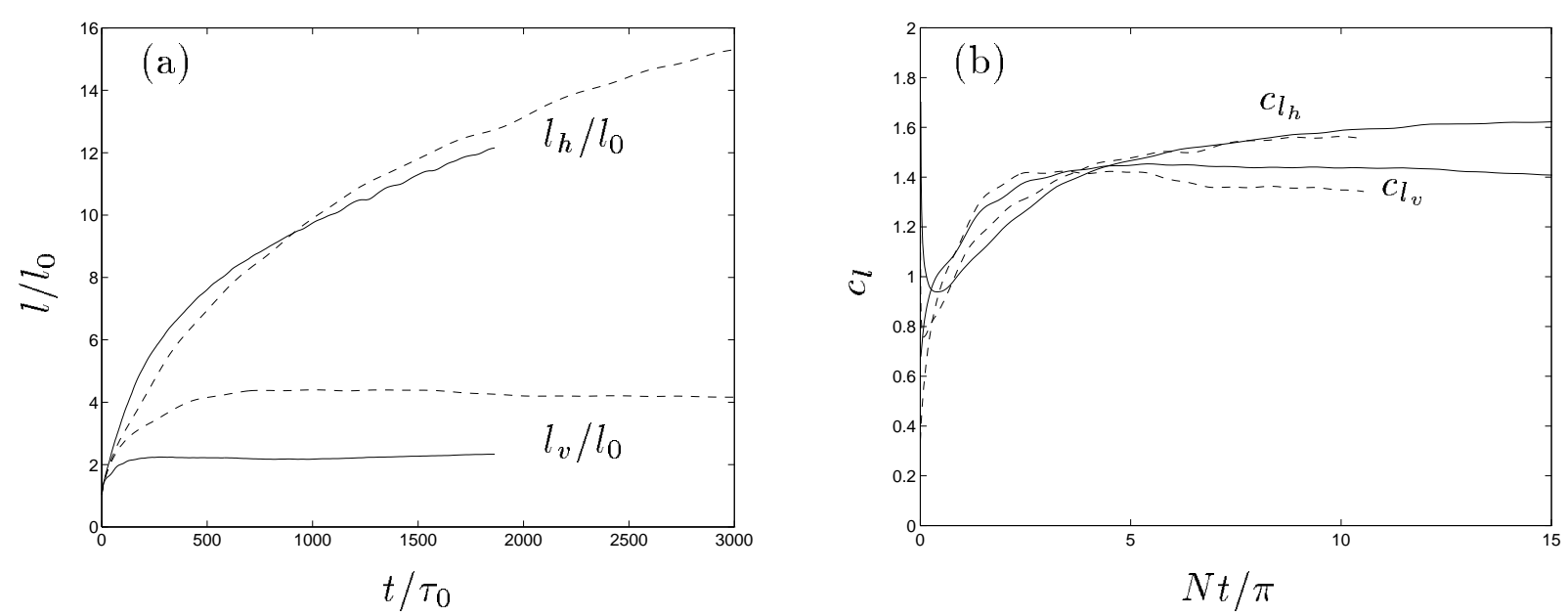

Figure 2. The integral length scales:,$- F_{0}=16.5 ; \cdots, F_{0}=93.2$; (a) timeevolution of the horizontal and vertical integral length scales of the total energy; (b) verification of the scaling relations given by (5.4) with $y=-2 / 5$ and $z=-1 / 10$.

isotropic velocity distribution and no density fluctuations. It is also possible that the similarity state which develops at small Froude numbers depends on the way in which the initial flow fields are initialized. Métais and Herring (1989) demonstrated by direct numerical simulations that the nature of the flow at small Froude numbers does depend on the relative state of the turbulence and wave field before entering the low Froude number regime. Some preliminary computations which we have performed verify that this is indeed the case; we intend to report on these findings at a later date.

\section{Acknowledgments}

Extensive discussions with R. S. Rogallo are gratefully acknowledged, as are the helpful comments of G. K. Batchelor, J. J. Riley, and K. D. Squires. The simulations were performed on the NAS Intel hypercube using software developed by R. S. Rogallo and A. Wray while the author was a post-doctoral fellow at the Stanford/NASA Ames Center for Turbulence Research. A continuation of this research is being supported by the Hong Kong Research Grant Council.

\section{REFERENCES}

Batchelor, G. K., Canuto, V. M. \& Chasnov, J. R. 1992. Homogeneous buoyancygenerated turbulence. J. Fluid Mech., 235: 349-378.

Chasnov, J. R. 1994. Similarity states of passive scalar transport in isotropic turbulence. Phys. Fluids, 6: 1036-1051.

Chollet, J. P. \& Lesieur, M. 1981. Parameterization of small scales of three dimensional isotropic turbulence utilizing spectral closures. J. Atmos. Sci., 38: 2747-2757.

Corrsin, S. 1951. The decay of isotropic temperature fluctuations in an isotropic turbulence. J. of the Aeronautical Sciences, 18: 417-423. 
Kolmogorov, A. N. 1941. On degeneration of isotropic turbulence in an incompressible viscous liquid. Dokl. Akad. Nauk. SSSR, 31: 538-541.

Larcheveque, M., Chollet, J. P., Herring, J. R., Lesieur, M., Newman, G. R., \& Schertzer, D. 1980. Two-point closure applied to a passive scalar in decaying isotropic turbulence. Turbulent Shear Flows II, Springer Verlag.

MÉtais, O. \& Herring, J. R. 1989. Numerical simulations of freely evolving turbulence in stably stratified fluids. J. Fluid Mech., 202: 117-148.

Riley, J. J., Metcalfe, R. W. \& Weissmann, M. A. 1981. Direct numerical simulation of homogeneous turbulence in density-stratified fluids. Nonlinear Properties of Internal Waves (ed. B. J. West), AIP Conf. Proc., 76, 79-112.

Rogallo, R. S. 1981. Numerical experiments in homogeneous turbulence. NASA TM 81315 .

Saffman, P. G. 1967a. The large-scale structure of homogeneous turbulence. J. Fluid Mech., 27: 581-593.

Saffman, P. G. 1967b. Note on decay of homogeneous turbulence. Phys. Fluids, 10: 1349 . 